\title{
Pathways of Fatty Acid Metabolism in Human Platelets
}

\author{
Phin Cohen, Arie Derksen, and Hendrik van den Bosch \\ From the Biochemistry Department, University of Utrecht, Utrecht, Holland, \\ and the Department of Medicine, Harvard Medical School and the Peter \\ Bent Brigham Hospital, Boston, Massachusetts 02115
}

A B S T R A C T The metabolic fate of ${ }^{14} \mathrm{C}$-labeled fatty acids which have been incubated with human platelets, has been traced. The following has been shown. (a) Intact platelets have a considerable capacity to oxidize fatty acids. (b) When tracer amounts of four of the most common fatty acids in normal plasma were incubated with platelets, each showed a distinctive pattern of uptake among neutral lipids and phospholipids. With regard to the latter, it was shown that these distribution patterns were, in most cases, similar to those of the fatty acids found in natural platelet phospholipids. (c) By increasing the time of incubation or the amount of added oleic acid, the distribution of oleic acid uptake between lecithin and other phosphoglycerides was altered so that a larger share was incorporated into the latter. (d) The effects of added lysolecithin or lysoethanolamine phosphoglycerides on oleic acid incorporation into platelet phosphoglycerides are quite variable. At low concentrations, added lysolecithin functions chiefly as a reaction partner for oleic acid. Added adenosine triphosphate and CoASH augment the incorporation of oleic acid into lecithin over a wide range of added lysolecithin (12.5-500 $\mu$ moles/liter). At higher concentrations of added lysolecithin, in the absence of ATP and CoASH, oleic acid incorporation into lecithin is considerably reduced. Also, added lysolecithin and lysoethanolamine phosphoglycerides, in the absence of ATP and CoASH, are able, at certain concentrations, to stimulate oleic acid incorporation into all except the serine phosphoglycerides. (e) Platelets appear to have a de novo pathway for renewal of lecithin.

\section{INTRODUCTION}

In contrast to the numerous studies of $\mathrm{FA}-{ }^{14} \mathrm{C}$ incorporation ${ }^{1}$ into erythrocyte PL (1-5), there has been only

\footnotetext{
Received for publication 17 September 1968 and in revised form 4 August 1969.

${ }^{1}$ The following abbreviations are used in the text and illustrations: PL, phospholipids; PC, choline phosphoglycerides (lecithins); PE, ethanolamine phosphoglycerides;
}

one reported study of $\mathrm{FA}-{ }^{14} \mathrm{C}$ uptake by human platelets (6). This latter study was concerned mostly with FA synthesis and the patterns of incorporation of labeled acetate and palmitic acid into platelet lipids; there was no attempt to systematically study the mechanism for acylation of platelet phospholipid precursors.

In the present work, using virtually pure platelet suspensions, which by the ${ }^{51} \mathrm{Cr}$-labeling technique previously had been shown to have a linear, 8 day lifespan (7), studies were undertaken to determine: $(a)$ the patterns of incorporation of various $\mathrm{FA}-1{ }^{14} \mathrm{C}$ into major $\mathrm{PL}$ and $\mathrm{NL} ;(b)$ the effect of added LPC or LPE on oleic acid$1-{ }^{14} \mathrm{C}$ incorporation into PL (Lands pathway) $(2,8,9)$; and $(c)$ the possible existence of a de novo pathway such as that described by Kennedy and Weiss (10) for synthesis of PC.

The purpose of this report is to present findings related to these topics.

\section{METHODS}

\section{Preparation of platelet suspensions}

For each study, $500 \mathrm{ml}$ of whole blood was freshly drawn from each of two fasting donors using acid-citrate-dextrose (ACD) anticoagulant and a plastic bag system. ${ }^{2}$ The donors were all male and were paired for erythrocyte $A B O-R h$ compatibility. They were chosen at random from the registry of the Netherlands Red Cross. The blood was centrifuged at $200 \mathrm{~g}$ for $15 \mathrm{~min}$, after which the upper three-fourths of the platelet rich plasma (PRP) was delivered into a dry $300 \mathrm{ml}$ plastic transfer pack. Excess ACD, $1 \mathrm{ml}$ for each $6 \mathrm{ml}$ of PRP, was then added. Without addition of excess $\mathrm{ACD}$ at this point the platelets could not be resuspended after subsequent centrifugations (7).

The plasma-ACD mixture was centrifuged at $150 \mathrm{~g}$ for 10 min to sediment erythrocytes. The PRP was separated

PI, inositol phosphoglycerides; PS, serine phosphoglycerides; SPH, sphingomyelins; LPC, lysocholine phosphoglycerides (lysolecithins); LPE, lysoethanolamine phosphoglycerides; NL, neutral lipids; DG, diglycerides; TG, triglycerides, FA, fatty acids; $16: 0$, palmitic acid; $18: 0$, stearic acid; $18: 1$, oleic acid; $18: 2$, linoleic acid; G-3-P, glycero-3-phosphate; GPC, glycerophosphorylcholine.

${ }^{2}$ Baxter Laboratories, Inc., Morton Grove, Ill. 
from the erythrocytes and the centrifugation was repeated until the sediment was almost completely white. The PRP was then centrifuged at $1000 \mathrm{~g}$ for $30 \mathrm{~min}$ to sediment a platelet pellet. The latter was resuspended in a mixture of $19 \mathrm{ml}$ of $300 \mathrm{mOsm}$ potassium phosphate buffer, $\mathrm{pH} 7.4$ and $1 \mathrm{ml} \mathrm{ACD}$ solution, and recentrifuged at $1000 \mathrm{~g}$ for 30 min, after which the supernatant was decanted; the platelet pellet was then resuspended in $5 \mathrm{ml}$ of fresh wash medium and kept in a plastic bag until use.

The processing of platelets was done at $1^{\circ} \mathrm{C}$, and all incubations were started within $6 \mathrm{hr}$ of the phlebotomies. Leukocyte and erythrocyte counts were done by standard procedures, platelet counts by the method of Brecher and Cronkite (11), and protein determinations by the biuret method (12). An average platelet concentrate had $6 \times 10^{6}$ platelets and less than 500 erythrocytes and 50 leukocytes/ $\mathrm{mm}^{3} .1 \mathrm{mg}$ of platelet protein equaled approximately $5 \times 10^{8}$ platelets.

Sonicated platelets were prepared by exposing intact platelets, in a vessel chilled by ice, to $1 \mathrm{~min}$. of sonic vibration at 20,000 cycles $/ \mathrm{sec}^{8}$

\section{Preparation of radioactive substrates}

Labeled glycerol-2- ${ }^{3} \mathrm{H}(0.5 \mathrm{mc} / \mathrm{mmole})$ and $\mathrm{FA}-1-{ }^{14} \mathrm{C}$ $(16: 0,18: 0,18: 1$, and $18: 2)$ at specific activities ranging from 25 to $50 \mathrm{mc} / \mathrm{mmole}$, were purchased; ${ }^{4}$ the radiochemical purity of the former was checked by paper chromatography, and that of the latter by gas-liquid chromatography. The FA, after removal of organic solvents in a rotary evaporator (using a nitrogen atmosphere to prevent peroxidation of unsaturated FA) were sonicated to form a water-clear solution in $2-4 \mathrm{ml}$ of $0.135 \mathrm{M} \mathrm{KCl-0.03} \mathrm{M}$ Tris buffer $(300 \mathrm{mOsm})$ at $\mathrm{pH} 7.5$. Several of the studies with $18: 1$ were repeated using the potassium salt of the FA in $0.5 \%$ albumin solution; the results of these studies were nearly identical with those with the FA sonicates.

$1-\left[9,10-{ }^{3} \mathrm{H}\right]-18: 0-$ LPC was prepared by methods previously reported from this laboratory (13). On the day of use, accumulated $18: 0-{ }^{3} \mathrm{H}$ was removed by chromatography on a $1 \mathrm{~mm}$ layer of Silica Gel G, in a chloroform : methanol : water $(65: 35: 4, \mathrm{v} / \mathrm{v} / \mathrm{v})$ developing system. The band containing the labeled LPC was scraped and eluted in $25 \mathrm{ml}$ chloroform: methanol $(20: 80, \mathrm{v} / \mathrm{v})$. The eluate was dried in a rotary evaporator and the residue was sonicated to form a water-clear solution in $2 \mathrm{ml}$ of $\mathrm{KCl}$-Tris buffer.

A $10 \mu \mathrm{l}$ sample of all labeled substrates was taken for radioactivity measurement in $16 \mathrm{ml}$ of a scintillation mixture made by dissolving $5 \mathrm{~g}$ of 2,5-diphenoloxazole and $0.3 \mathrm{~g}$ of 1,4-bis-2 (methyl-5-phenoloxazolyl) benzene, in $1000 \mathrm{ml}$ of toluene. The samples were counted in an automatic Packard Tri-Carb liquid scintillation spectrometer, and quenching corrections were made by the channel ratio method (14).

\section{Preparation of nonradioactive substrates}

LPC and LPE were prepared from rat liver PL. The lipid extracts of four rat livers were separated on a preparative scale using $20 \times 20 \mathrm{~cm}$ glass plates coated with a $1 \mathrm{~mm}$ layer of Silica Gel $\mathrm{H}$ and developed in chloroform : methanol: $18 \mathrm{~N}$ acetic acid: water $(50: 30: 8: 4, \mathrm{v} / \mathrm{v} / \mathrm{v} / \mathrm{v})$. The $\mathrm{PC}$ and $\mathrm{PE}$ bands were scraped into separate columns and eluted by chloroform: methanol $(1: 1, \mathrm{v} / \mathrm{v})$. Each eluate was subjected to snake venom phospholipase (EC 3.1.1.4) degra-

\section{${ }^{8}$ Branson Europa N.V., Loosdrecht, Holland.}

"The Radiochemical Centre, Amersham, England. dation (15) and the resulting lysocompounds were isolated by thin-layer chromatography using Silica Gel G and a chloroform: methanol: water $(65: 35: 4, \mathrm{v} / \mathrm{v} / \mathrm{v})$ developing system. The LPC and LPE were eluted from the silica gel with chloroform: methanol $(20: 80, \mathrm{v} / \mathrm{v})$. The eluate was taken to dryness and the lipid residue was redissolved in $10 \mathrm{ml}$ of chloroform: methanol mixture before measurement of the phosphorus concentration by the method of Bartlett (16).

In addition, 1-16:0-LPC was synthesized by the method of Baer and Buchnea (17). On the day of use, water-clear sonicates of the lysocompounds and nonradioactive FA were prepared in $\mathrm{KCl}$-Tris buffer.

Adenosine triphosphate (ATP) ${ }^{5}$ and $\mathrm{CoASH}^{\circ}$ were prepared as a solution of $\mathrm{pH} 7.4$, having $50 \mathrm{~mm}$ and $0.3 \mathrm{~mm}$ concentration of ATP and $\mathrm{CoASH}$, respectively; glycerol, and glycero-3-phosphate, ${ }^{8}$ as $10 \mathrm{~mm}$ solutions.

\section{Incubation procedures}

All incubations were done in an automatic shaking water bath at $37^{\circ} \mathrm{C}$. The final volume in each case was adjusted to $2 \mathrm{ml}$ with $\mathrm{KCl}$-Tris buffer. The $\mathrm{pH}$ was 7.3 at the start and 7.1 at the end of a $3 \mathrm{hr}$ incubation. The glucose concentration, measured by the glucose oxidase method (18) was 5-6 mmoles/liter in all incubations, the glucose deriving entirely from the ACD used in processing the blood. Platelet clumping was carefully watched for; none was observed during or at the end of any incubation.

Reactions were stopped by the addition of $5 \mathrm{ml}$ of methanol and $2 \mathrm{ml}$ of chloroform which gave a single phase; lipids were then extracted by the Bligh and Dyer (19) method, with two additional washes of the aqueousmethanol layer with chloroform.

After evaporation of the combined chloroform extracts, the residues were prepared for chromatographic procedures by the addition of $250 \mu 1$ of chloroform: methanol $(9: 1$, $\mathrm{v} / \mathrm{v})$.

In some studies, parallel incubations were extracted by the single phase method of Ways and Hanahan (20); in others the platelets were separated from the incubation mixture (by sedimentation at $4500 \mathrm{~g}$ for $30 \mathrm{~min}$ at $4^{\circ} \mathrm{C}$ ) before extraction. In both cases, results were nearly identical with those using the simpler and faster Bligh and Dyer method. In the sedimentation studies, it was shown that neither iodine-stainable, nor ${ }^{14} \mathrm{C}$-labeled lipid was present in the supernatant.

Thin-layer chromatography and assay of radioactivity in isolated PL and NL

A. Single dimension for separation of major $P L$. This procedure was a combination of methods described by Skipski, Peterson, and Barclay (21) and Hofmann (22). Glass plates, $5 \times 20 \mathrm{~cm}$, were covered with a $0.5 \mathrm{~mm}$ film of Silica Gel $\mathrm{H}$ impregnated with $3 \%\left(\mathrm{NH}_{4}\right)_{3} \mathrm{PO}_{4}(\mathrm{w} / \mathrm{w})$ and allowed to dry before activation at $110^{\circ} \mathrm{C}$ for $3 \mathrm{hr}$. Without $\left(\mathrm{NH}_{4}\right)_{3} \mathrm{PO}_{4}$ impregnation of Silica Gel H, PI, and PS migrated together as a homogeneous spot. The total lipid extract from an incubation was applied in six equal spots and chromatograms were developed in chloroform: methanol: $18 \mathrm{~N}$ acetic acid: water $(50: 30: 8: 4, \mathrm{v} / \mathrm{v} / \mathrm{v} / \mathrm{v})$ to within $3 \mathrm{~cm}$ of the top of the plate. After drying in air for

${ }^{5}$ Fluka AG, Switzerland.

' Pabst Laboratories, Milwaukee, Wis.

${ }^{7}$ E. Merck A.G., Darmstadt, Germany.

${ }^{8}$ Fluka AG, Switzerland. 


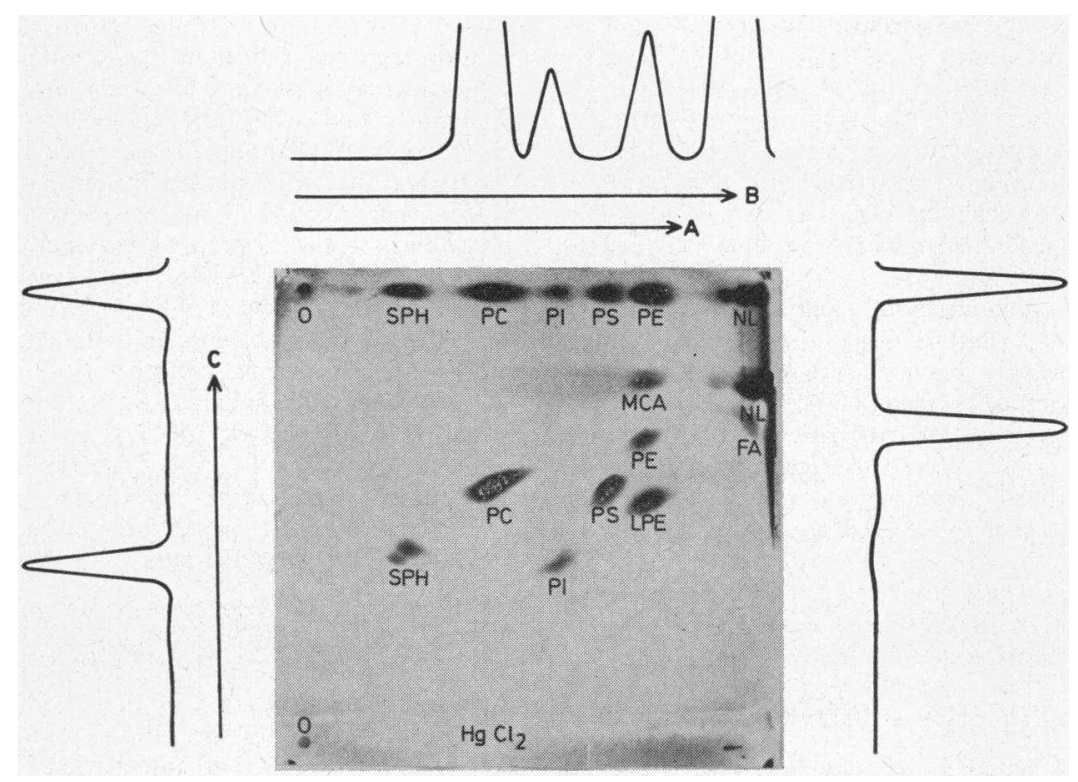

FIGURE 1 Photograph of iodine-stained thin-layer chromatogram of platelet lipid extract from an incubation of $4 \mathrm{mg}$ of platelet protein with $30 \mathrm{~m} \mu$ moles of $1-{ }^{14} \mathrm{C}$-labeled 16:0 for $3 \mathrm{hr}$. The arrows indicate the direction and limits of development with the three solvent systems. The lipid track at the top of the plate was developed only in one dimension. It shows the typical separation of $\mathrm{PL}$ that is achieved on $\left(\mathrm{NH}_{4}\right)_{3} \mathrm{PO}_{4}$ impregnated Silica Gel $\mathrm{H}$, using $(A)$ chloroform: methanol: $18 \mathrm{~N}$ acetic: water $(50: 30: 8: 4, \mathrm{v} / \mathrm{v} / \mathrm{v} / \mathrm{v})$, and $(B)$ a postrun in dry, distilled ether. The lipid track at the bottom of the plate was sprayed with $\mathrm{HgCl}_{2}$ and developed in $(C)$ chloroform: methanol: $13.5 \mathrm{~N}$ ammonia: water $(70: 30: 0.5: 4, \mathrm{v} / \mathrm{v} / \mathrm{v} / \mathrm{v})$; it shows a clear separation of all major PL, including the diacyl and plasmalogen forms of $\mathrm{PE}$, the latter being represented by LPE and the mercurichloride derivative (MCA) of the 1-position alkenyl group. Only the PC, PI, and PE showed appreciable amounts of radioactivity after scans of both dimensions of the chromatogram. The upper scan is of the first dimension; those on the left and right are, respectively, of the PI and PE tracks to include first and second dimension spots.

15 min, a postrun was done in dry, distilled ether, to carry free, excess $\mathrm{FA}-1-{ }^{14} \mathrm{C}$ to the top of the plate. The PL were marked after exposing the plate to iodine vapor, and identified by comparison with pure reference compounds. After evaporation of the iodine in air, the bands were scraped into a vial containing thixotropic gel $^{9}$ into which the scintillation fluid described above, was blended. The samples were counted as previously described.

$B$. Two-dimensional separation of PE plasmalogens from other major $P L$ (Fig. 1). This procedure was a modification of the method of Owens $(23) .20 \times 20 \mathrm{~cm}\left(\mathrm{NH}_{4}\right)_{3} \mathrm{PO}_{4-}$ impregnated Silica $\mathrm{Gel} \mathrm{H}$ plates were prepared and activated as in A (above). From a $250 \mu \mathrm{l}$ chloroform: methanol lipid extract of an incubation, two $50 \mu \mathrm{l}$ spots were applied 2 and $3 \mathrm{~cm}$ from the left- and right-hand edges of the plate, respectively. The plate was developed in the first dimension as described in A. After evaporation of the developing solvents in air, the residual acetic acid was neutralized by supporting the plate for $3 \mathrm{~min}$ in a closed tank which was saturated with $\mathrm{NH}_{3}$ vapor. The $\mathrm{NH}_{3}$ was then completely removed under a stream of cold air, and the left-hand lipid track was sprayed with a solution of $5 \mathrm{~mm} \mathrm{HgCl}_{2}$ in water while the remainder of the chromatogram was screened. The plate was reactivated in a vacuum desiccator at room temperature for $1 \mathrm{hr}$ and the $\mathrm{HgCl}_{2}$-treated lipid track was de-

${ }^{\bullet}$ Thixcin, Packard Laboratories, Brussels, Belgium. veloped in the second dimension with chloroform: methanol: $13.5 \mathrm{~N}$ ammonia: water $(70: 30: 0.5: 4, \mathrm{v} / \mathrm{v} / \mathrm{v} / \mathrm{v})$. The plates were then dried and scanned in both dimensions using a proportional gas-flow counter with automatic count integration $;^{10}$ the lipids were identified, scraped off, and assayed for radioactivity as in $A$.

C. Two-dimensional separation of NL (Fig. 2). $20 \times 20$ $\mathrm{cm}$ plates were covered with a $0.7 \mathrm{~mm}$ thick Silica Gel G layer, dried in air, and activated at $110^{\circ} \mathrm{C}$ for $2 \mathrm{hr}$. The $150 \mu \mathrm{l}$ of extract not used in section $\mathrm{B}$, along with an internal reference mixture of 1,2- and 1,3-DG, was spotted $2 \mathrm{~cm}$ from the right-hand edge of the plate. Development in the first dimension was with dry, distilled ether: petroleum ether $\left(\right.$ bp $\left.40^{\circ}-60^{\circ} \mathrm{C}\right): 18 \mathrm{~N}$ acetic acid $(60: 40: 0.5, \mathrm{v} / \mathrm{v} / \mathrm{v})$ to $0.5 \mathrm{~cm}$ from the top of the plate. After evaporation of the solvents under a stream of cold air, the plate was developed in the second dimension with hexane: ether $(80: 20$, $\mathrm{v} / \mathrm{v})$. The plates were then scanned in both dimensions, and the DG and TG spots were detected, scraped off, and assayed for radioactivity, as in section A. The FA spot was well separated from the other lipids and was not "seen" on the scans of the lipid tracks containing the DG and TG spots.

The tivo-dimensional techniques were used only for the studies in which the patterns of incorporation of

${ }^{10}$ Berthold, Wildbad, Germany. 


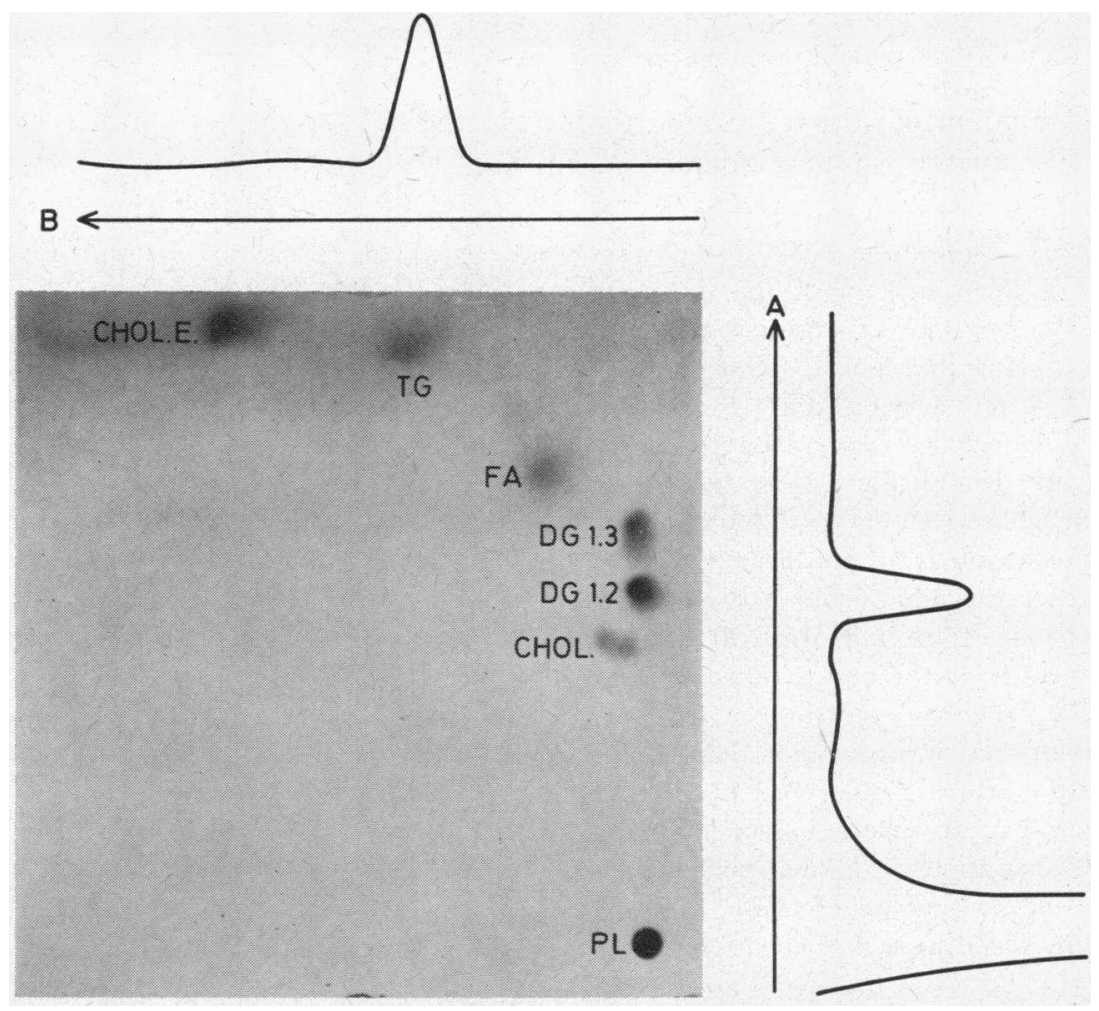

FIGURE 2 Photograph of iodine-stained thin-layer chromatogram of platelet lipid extract from an incubation of $4 \mathrm{mg}$ of platelet protein with $30 \mathrm{~m} \mu$ moles of $1-{ }^{14} \mathrm{C}-$ labeled $16: 0 \mathrm{for} 3 \mathrm{hr}$. The arrows indicate the direction and limits of development with the two solvent systems; $(A)$ dry, distilled ether: petroleum ether (bp 40-60):18 $\mathrm{N}$ acetic acid $(60: 40: 0.5, \mathrm{v} / \mathrm{v} / \mathrm{v})$ and $(B)$ hexane: ether $(80: 20, \mathrm{v} / \mathrm{v})$. Only the DG and TG showed appreciable accumulation of radioactivity after scans of both dimensions of the chromatogram. The FA spot was well separated from the other lipids and was not "seen" on the scan of the first dimension. CHOL= cholesterol; CHOL $\mathrm{E}=$ cholesterol esters.

various $\mathrm{PL}$ and $\mathrm{NL}$ of platelets, were determined (see Tables I and II). For the remaining studies the singledimensional system described in section $A$ was used since, with intact platelets, the labeling of $\mathrm{PE}$ plasmalogens could, in all cases, be ignored.

Assay of radioactivity in experiments using ${ }^{3} \mathrm{H}$ labeled substrates (1-18: O-LPC or glycerol)

After identification, the silica gel containing the PC band was scraped into a Pasteur pipette microcolumn. The lipid material was eluted with $100 \mathrm{ml}$ of chloroform: methanol $(20: 80, \mathrm{v} / \mathrm{v})$ which ran through over a period of $6-12 \mathrm{hr}$. The solvents were then removed in a rotary evaporator, and the lipid residue was dissolved in $1 \mathrm{ml}$ of methanol from which a $200 \mu \mathrm{l}$ aliquot was taken for radioactivity measurement, using an external standard for quenching corrections.

In some experiments on the uptake of glycerol- $2{ }^{8} \mathrm{H}$, the isolated PC was eluted from the silica gel and then subjected to alkaline hydrolysis by the method of Benson and Maruo (24). The water-soluble products were separated by descending chromatography on Whatman paper in a propanol : ammonia: water $(6: 3: 1, \mathrm{v} / \mathrm{v} / \mathrm{v})$ developing system, and identified by comparison with pure reference compounds, after staining of the chromatogram with the periodate-
Schiff reagent. The glycerol, G-3-P, and GPC spots were then cut out and eluted with $50 \mathrm{ml}$ of a water: methanol $(4: 1, \mathrm{v} / \mathrm{v})$ solution. The latter was evaporated and the residue dissolved in $200 \mu \mathrm{l}$ of a water : methanol solution, in preparation for assay of radioactivity.

\section{RESULTS}

\section{FA oxidation}

The amount of recoverable radioactivity in the chloroform layer of the Bligh and Dyer extraction decreased linearly during a $3 \mathrm{hr}$ incubation of $30 \mathrm{~m} \mu$ moles of 18:1 with $4 \mathrm{mg}$ of platelet protein as intact platelets; however, there was virtually no change in the very low content of radioactivity in the aqueous-methanol layer (Fig. 3). FA oxidation was shown to have a lag phase of 25-30 min, after which it became linear during a 4 $\mathrm{hr}$ incubation.

When the radioactivity trapped in hyamine as ${ }^{14} \mathrm{CO}_{2}$ (25) was added to that measured in both layers of the Bligh and Dyer extraction, 90-95\% of the added 18:1$1{ }^{14} \mathrm{C}$ could be accounted for after a $3 \mathrm{hr}$ incubation. 
Sonication abolished FA oxidation and resulted in an even higher recovery (98-99\%) of added radioactivity.

Effect of time and amount of added 18: 1 on the distribution of its uptake among major PL (Fig. 4)

With $4 \mathrm{mg}$ of platelet protein and $30 \mathrm{~m} \mu$ moles of added 18:1 the rate of incorporation of this FA into $\mathrm{PE}, \mathrm{PI}$, and PS was linear for $3 \mathrm{hr}$, whereas that into PC showed a more rapid initial phase followed by a gradually decreasing rate of incorporation.

In the range of 25-200 m $\mu$ moles of added $18: 1$, the amount incorporated into PC during a $3 \mathrm{hr}$ incubation period increased linearly; between 200 and 400 mumoles the linearity gradually plateaued. An apparent maximum level of incorporation was reached at 600 or $700 \mathrm{~m} \mu$ moles. On the other hand, the incorporation of 18:1 into the other PL remained linear over the entire range of concentrations of added 18:1.

Subsequent studies of FA incorporation into lipids employed 3-hr incubation times and $4 \mathrm{mg}$ of platelet protein per incubation. Except where indicated otherwise, 30 m $\mu$ moles FA ( 5 m $\mu$ moles $1{ }^{14} \mathrm{C}$ material plus 25 m $\mu$ moles of carrier) and $5 \mu$ moles of ATP and 0.3 $\mu$ moles of $\mathrm{CoASH}$ were added to each incubation.

Patterns of incorporation of four FA into major PL (Tables I and II)

The unequal incorporation of added $18: 1-1{ }^{14} \mathrm{C}$ among platelet PL, and particularly the relatively heavy labeling of PI and nearly absent labeling of PS, raised the question of whether a similar distribution pattern would obtain with other FA-1 $-{ }^{14} \mathrm{C}$. Thus, to add to the data shown in Fig. 4, we studied the patterns of incorporation of the four most common FA of human plasma into all of the major PL of the platelet. The

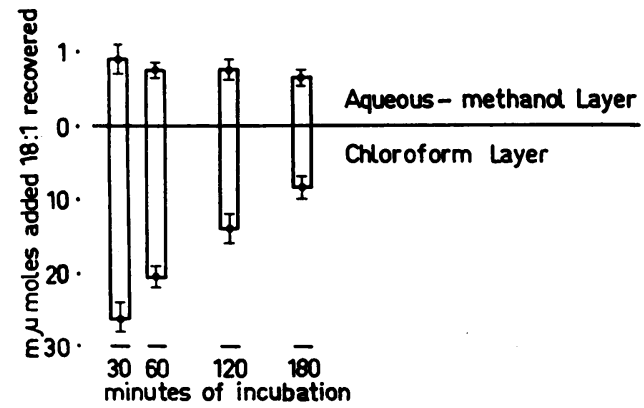

Figure 3 Recovery of radioactivity in Bligh and Dyer extraction after incubation of intact platelets with 30 mumoles of $1-{ }^{14} \mathrm{C}$-labeled $18: 1$ for varying time intervals. The ordinate shows the mumoles of 18:1 accounted for in the upper (aqueous methanol) and lower (chloroform) layers; the abscissa, the time of incubation. Note that the scale on the ordinate is different for the two layers. The data shown are the means $\pm \mathbf{S D}$ of four experiments.

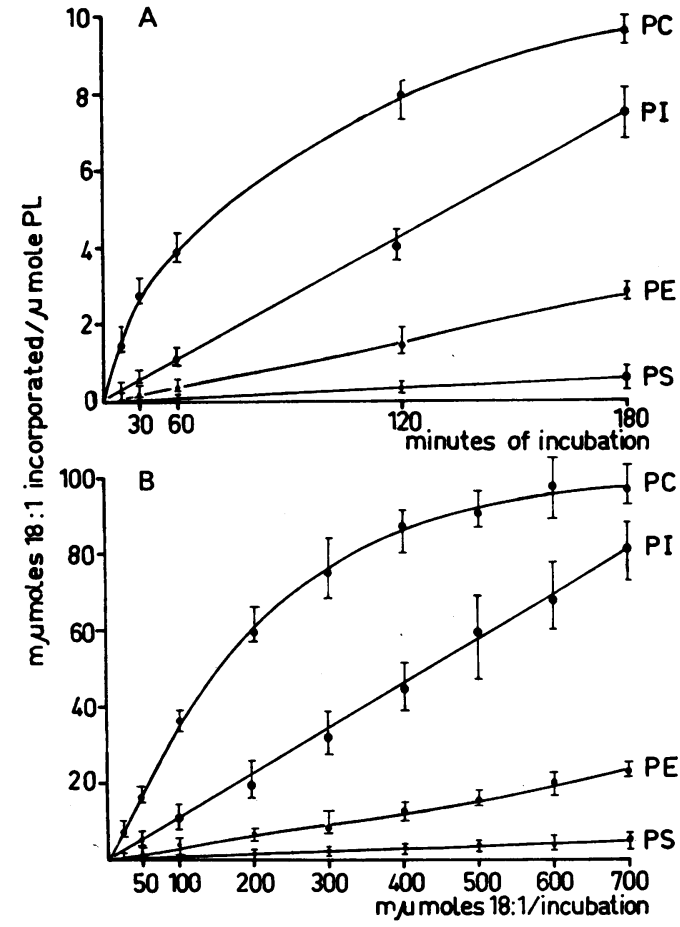

Figure 4 Effect of varying the time of incubation or the amount of added 18:1 on its incorporation into four major platelet PL. The ordinate shows the mumoles 18:1 incorporated per $\mu$ mole PL; the abscissa the time of incubation (A), or the amount of added 18:1 (B). Results shown are means \pm SD of four separate studies. See legend of Table I for method of calculating specific activities of FA incorporation into various $\mathrm{PL}$.

data indicate that each labeled FA has a distinctly different pattern of incorporation into platelet PL (Table I). For example, using intact platelets and considering only the PC, 16:0 showed the highest rate of incorporation of the four FA. On the other hand the uptake of 18:0 by PC was by far the lowest of the FA studied. However, diacyl PE, PS, and PI showed a definite relative affinity for incorporating 18:0, in comparison with the other FA. In contrast to the differences between the saturated FA, the patterns of incorporation of $18: 1$ and 18: 2 were remarkably similar.

Lands, Blank, Nutter, and Privett, in studies with rat liver microsomes, have found that acyltransferase activities measured in vitro have a considerable predictive value in terms of the ultimate distribution of $F A$ in glycerolipids in vivo (26). To determine whether a similar predictive value could also apply to in vitro studies with human platelets we compared the results of the metabolic studies with our quantitative data on the PL and FA composition of platelets that were derived from the same donor pool (27). 
TABLE I

Incorporation of $F A-1{ }^{14} C$ into Human Platelet $P L$ In Vitro

\begin{tabular}{|c|c|c|c|c|c|c|c|c|}
\hline & \multicolumn{2}{|c|}{$16: 0$} & \multicolumn{2}{|c|}{$18: 0$} & \multicolumn{2}{|c|}{$18: 1$} & \multicolumn{2}{|c|}{$18: 2$} \\
\hline & I & $\mathbf{S}$ & I & $\mathbf{S}$ & I & $\mathbf{S}$ & I & $\mathbf{S}$ \\
\hline & \multicolumn{8}{|c|}{ mumoles $F A$ incorporated/umole $P L^{*}$} \\
\hline PC & $11.3 \pm 0.8$ & $9.3 \pm 0.7$ & $6.8 \pm 0.7$ & $2.7 \pm 0.7$ & $9.7 \pm 0.5$ & $13.4 \pm 0.6$ & $9.9 \pm 1.5$ & $14.0 \pm 1.2$ \\
\hline Diacyl PE & $2.8 \pm 0.1$ & $2.0 \pm 0.1$ & $12.8 \pm 0.6$ & $5.6 \pm 0.2$ & $3.2 \pm 0.2$ & $6.1 \pm 0.5$ & $2.6 \pm 0.2$ & $4.1 \pm 0.6$ \\
\hline Plasmalogen PE & $0.3 \pm 0.06$ & $0.7 \pm 0.15$ & $0.4 \pm 0.1$ & $0.3 \pm 0.06$ & $0.3 \pm 0.06$ & $2.8 \pm 0.2$ & $0.3 \pm 0.06$ & $0.6 \pm 0.1$ \\
\hline PS & $0.4 \pm 0.06$ & $2.7 \pm 0.7$ & $2.4 \pm 0.2$ & $4.4 \pm 0.2$ & $0.7 \pm 0.1$ & $1.1 \pm 0.1$ & $0.8 \pm 0.2$ & $1.7 \pm 0.2$ \\
\hline PI & $3.6 \pm 0.5$ & $4.1 \pm 0.9$ & $28.3 \pm 1.6$ & $13.1 \pm 0.2$ & $7.9 \pm 0.8$ & $6.7 \pm 0.1$ & $7.3 \pm 0.3$ & $5.7 \pm 0.3$ \\
\hline
\end{tabular}

Each reaction mixture contained $4 \mathrm{mg}$ platelet protein, $5 \mu$ moles ATP, $0.3 \mu$ mole CoASH, and $30 \mathrm{~m} \mu \mathrm{moles}$ FA (1-14C material plus carrier). All incubations were for $3 \mathrm{hr}$. See Methods (and Fig. 1) for a description of the two-dimensional system used for separating the major PL, and for procedures used to assay radioactivity. The data are the means $\pm S D$ of four separate studies using intact (I) or sonicated (S) platelets in parallel incubations. A single experiment was performed in which each of the four FA was incubated with intact platelets in the absence of ATP and CoA (results not shown). The observed patterns of incorporation into the major PL were similar to those found with ATP and CoASH addition.

* The PL composition of the incubations was calculated from previous studies (27) using platelets derived from donors selected at random from the same donor list. Thus, each incubation was calculated to have had the following PL composition (mean values listed in micromoles) PC, 0.412 ; diacyl PE, 0.140 ; plasmalogen PE, 0.145 ; PS, 0.091 ; PI, 0.038 .

Table II A shows, for the three PL which were most heavily labeled in the incubation studies, the specific activity of tracer (exogenous) FA as opposed to the relative abundance of the corresponding nonradioactive (endogenous) FA. For the PE, the relative levels of incorporation of tracer amounts of FA increased in the same order as the relative amounts of endogenous FA in the PE fraction $(18: 2<16: 0<18: 1<18: 0)$. This held true for the PC and PI, with exceptions of $18: 2$ for $\mathrm{PC}$ and PI, and 16:0 for PI.
For a more direct comparison of the relative amounts of "exogenous" and "endogenous" FA in the three PL, PC: PE and PC: PI ratios were calculated (Table II B). With two exceptions, the PC:PI ratios for 16:0 and $18: 2$, the ratios of exogenous and endogenous FA distribution agreed rather closely.

Effect of mechanical disruption of platelets on incorporation of FA into PL

With intact platelets the transport-permeability behavior of a particular FA and the anatomical sequestra-

TABLE II

Relative Levels of Incorporation of Tracer Amounts of Four FA-1-14C into Three Human Platelet $P L^{*}$ as Compared with the Concentration of the Corresponding FA in the Natural PLף

\begin{tabular}{|c|c|c|c|c|c|c|c|}
\hline & FA & Incorporated* & Endogenous $\ddagger$ & Incorporated* & Endogenous $f$ & Incorporated* & Endogenous $\ddagger$ \\
\hline \multirow{6}{*}{ A. } & & \multicolumn{2}{|c|}{ 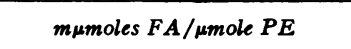 } & \multicolumn{2}{|c|}{ mumoles $F A /$ mmole $P C$} & \multicolumn{2}{|c|}{ mumoles FA/umole PI } \\
\hline & $16: 0$ & 2.8 & 116 & 11.3 & 554 & 3.6 & 34 \\
\hline & $18: 0$ & 12.8 & 496 & 6.8 & 300 & 28.3 & 846 \\
\hline & $18: 1$ & 3.2 & 244 & 9.7 & 496 & 7.9 & 318 \\
\hline & $18: 2$ & 2.6 & 82 & 9.9 & 236 & 7.3 & 50 \\
\hline & & \multicolumn{2}{|c|}{$\begin{array}{c}\text { Incorporated } \\
\text { PC: PE }\end{array}$} & $\begin{array}{c}\text { Endogenous } \\
\text { PC:PE }\end{array}$ & $\begin{array}{c}\text { Incorporated } \\
\text { PC: PI }\end{array}$ & $\begin{array}{c}\text { Endogenous } \\
\text { PC:PI }\end{array}$ & \\
\hline \multicolumn{8}{|l|}{ B. } \\
\hline & $16: 0$ & & 4.0 & 4.8 & 3.14 & 16.3 & \\
\hline & $18: 0$ & & 0.53 & 0.60 & 0.24 & 0.35 & \\
\hline & $18: 1$ & & 3.0 & 2.0 & 1.23 & 1.5 & \\
\hline & $18: 2$ & & 3.8 & 2.9 & 1.36 & 4.7 & \\
\hline
\end{tabular}

The data in A have been calculated to show the ratios of the amounts of tracer (incorporated) or of natural (endogenous) FA in two PL pairs (PC:PE and PC:PI). For example, for 16:0 there are $554 \mathrm{~m} \mu \mathrm{moles} / \mu \mathrm{mole} \mathrm{PC}$ and $116 \mathrm{~m} \mu \mathrm{moles} / \mu$ mole $\mathrm{PE}$ in circulating platelets; the ratio of these numbers is 4.8 , while that calculated from the in vitro uptake of $16: 0-1-{ }^{14} \mathrm{C}$ into these $\mathrm{PL}$ is 4.0 . The data listed are the mean values of four experiments for the incorporation studies; and three for the composition studies.

* From $30 \mathrm{~m} \mu$ moles added FA (from the data in Table I).

$\ddagger$ Data from our recently reported work on the PL and FA composition of human platelets (27). 
tion of certain acyltransferase systems could influence the distribution of FA among PL. It is possible that in disrupted cells the former could be facilitated, and the latter unmasked. Thus, in parallel with the studies of intact platelets, incubations were done with sonicated platelets.

That this disruption could affect intracellular structures as well as the plasmalemmal membrane was shown by the loss of FA oxidative capacity, probably secondary to the damaged mitochondria, in platelets so treated. Sonication of the platelets raised the uptake of the unsaturated FA into both $\mathrm{PC}$ and $\mathrm{PE}$, but had an opposite effect on the corresponding uptakes of saturated FA (Table I). On the other hand, the incorporation of all FA into PS was substantially augmented by sonication; the opposite effect, except with 16:0, was found with PI. Under the same conditions, there was increased uptake of 16:0 and 18:1 into the PE plasmalogens.

\section{Incorporation of FA into NL}

Mammalian NL and PL isolated from the same tissue are known to have different FA compositions (28). As a rule, the NL fraction has a slightly higher 16:0 content and a much higher 18:1 content than the PL fraction. On the other hand, the PL have a relatively higher content of $18: 0$. We have no data pertaining to the FA composition of platelet NL, thus precluding the same type of comparison between in vitro incorporation studies and FA composition of the natural lipid, as was done with the PL. Nevertheless, we suspect that the in vitro studies will not have the predictive value in terms of the ultimate distribution of the $\mathrm{FA}$ in $\mathrm{NL}$ in vivo.

The incorporation of 16:0 and 18:0 into DG and TG was two to three times higher than was found with 18:1 and 18:2. Only in the case of $18: 2$ did sonication

TABLE III

Incorporation of Equimolar Tracer Amounts of $1-\left[9,10^{3} \mathrm{H}\right] 18: 0-L P C$ and $18: 1-1{ }^{14} \mathrm{C}$ into the $P C$ of Intact Platelets

\begin{tabular}{|c|c|c|c|}
\hline LPC & $18: 1$ & LPC & $18: 1$ \\
\hline \multicolumn{2}{|c|}{ mumoles/incubation } & \multicolumn{2}{|c|}{ mumoles incorporated/umole $P C$} \\
\hline 15 & 15 & 7.3 & 6.9 \\
\hline 15 & 15 & 7.6 & 7.1 \\
\hline 30 & 30 & 12.7 & 12.2 \\
\hline 30 & 30 & 15.9 & 15.4 \\
\hline
\end{tabular}

The data shown are results from two experiments in which platelet suspensions containing $4 \mathrm{mg}$ of platelet protein, 5 $\mu$ moles of ATP, and $0.3 \mu$ moles of $\mathrm{CoASH}$ were incubated for $3 \mathrm{hr}$ with equimolar amounts (15 or $30 \mathrm{~m} \mu$ moles) of $1-\left[9,10^{3} \mathrm{H}\right]$ 18:0-LPC and $18: 1-1-{ }^{14} \mathrm{C}$. See methods for a description of procedures for identifying and assaying radioactivity in PC.

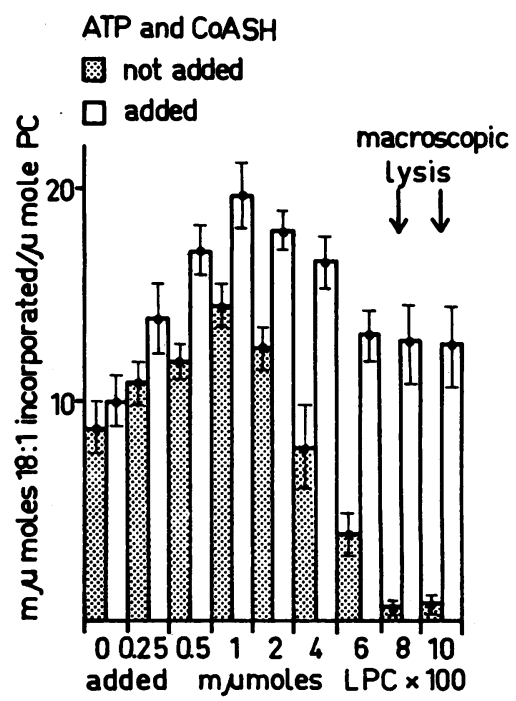

FIgure 5 The effect of added LPC on the incorporation of $1-{ }^{14} \mathrm{C}$-labeled 18:1 into $\mathrm{PC}$, with and without added ATP and CoASH. The ordinate shows the mumoles 18:1 incorporated per $\mu$ mole PC; the abscissa the m $\mu$ moles of $1-16: 0$. LPC added. When used, ATP and CoASH were added in amounts of 5 and $0.3 \mu$ moles, respectively. The results shown are the means $\pm \mathrm{SD}$ of three experiments.

of the platelets raise the incorporation into DG, whereas in all cases it virtually abolished uptake into TG.

Studies of 18: 1 incorporation into PL by lands pathway

The acylation of lysocompounds is an important mechanism for renewal of $\mathrm{PL}(2,8,9,26,29)$. It provides a means for the partial, as opposed to the total, synthesis of PL, and in addition, can be viewed as a system of defense against the buildup of lytic levels of lysocompounds. Both of these considerations, economy of PL renewal and self-defense against physiological lytic agents, are obviously of importance to blood cells. The problem in FA incorporation studies with added lysocompounds is to distinguish between their acyl acceptor function and their ability to damage membranes, resulting in a physicochemical facilitation of acyltransferase reactions. We have, therefore, studied the effects of various amounts of added lysocompounds on 18:1 incorporation into four of the major PL of the platelet.

Effect of small amounts of added LPC: double label experiments (Table III). When 15 or $30 \mathrm{~m} \mu$ moles of both $18: 1-1{ }^{14} \mathrm{C}$ and $1-\left[9,10^{3} \mathrm{H}\right] 18: 0-$ LPC were added to a reaction mixture, these substrates were incorporated into $\mathrm{PC}$ in nearly equimolar amounts. In each case, there was a slightly greater uptake of the LPC, as opposed to the FA, into the PC.

Effect of added ATP and CoASH over a wide range of added LPC (Fig. 5). Up to a concentration of 100 
$\mathbf{m} \mu$ moles of added LPC per incubation, there was a linear increase in 18:1 incorporation into $\mathrm{PC}$, which was $27-37 \%$ higher in the presence of added ATP and CoASH. When larger amounts of LPC were added, incorporation of 18:1 into PC declined from the peak value. This decline was considerably less marked with added ATP and CoASH, particularly at the highest concentrations of LPC, which were able to induce macroscopic lysis of platelets.

Effect of added LPC or LPE on patterns of incorporation into major $P L$ (Fig. 6). In the absence of added ATP and CoASH, added LPC and LPE were able to augment 18:1 incorporation into $\mathrm{PC}, \mathrm{PE}$, and $\mathrm{PI}$, but not PS. This effect was particularly striking in the experiments with LPE.

When ATP and CoASH were added the stimulatory effect of LPC and LPE was limited to specific reaction partners (LPE for PE; LPC for PC) whereas incorporation into other PL was either not stimulated at all, or was, in fact, less than the control value (curves not shown).

Studies of de novo PL synthesis by platelets

The acyl acceptor of de novo pathways for PL synthesis, G-3-P, is known to be a poor penetrator of in- tact cells. However, not all membranous structures are similarly affected. It is known that external G-3-P readily penetrates isolated liver mitochondria (30) and thus is able to function as an acyl acceptor $(31,32)$ perhaps by replenishing endogenous G-3-P which has been lost during the isolation procedure (32).

Since platelets isolated by our technique are known to lose only small amounts of $\mathrm{K}$ during a $1 \mathrm{hr}$ incubation at $37^{\circ} \mathrm{C}(33)$, and have a linear rate of destruction and a normal lifespan if returned to the circulation (7), it is a reasonable assumption that their plasmalemmal barriers are intact. Thus, it was not an unexpected finding that G-3-P, added in amounts varying from 100 to 1000 $\mathrm{m} \mu$ moles per incubation did not significantly increase incorporation of 18:1 into PC. Sonication of the platelets achieved no better results; however, it is possible that the operation of the multi-enzyme de novo synthesis system, unlike the less complicated Lands pathway, is more dependent upon an intact architectural platform for optimal activity.

Therefore, the permeability barrier was bypassed by using added glycerol, which readily penetrates most mammalian membranes, as a probe for the presence of a de novo synthesis pathway. In demonstrating its uptake

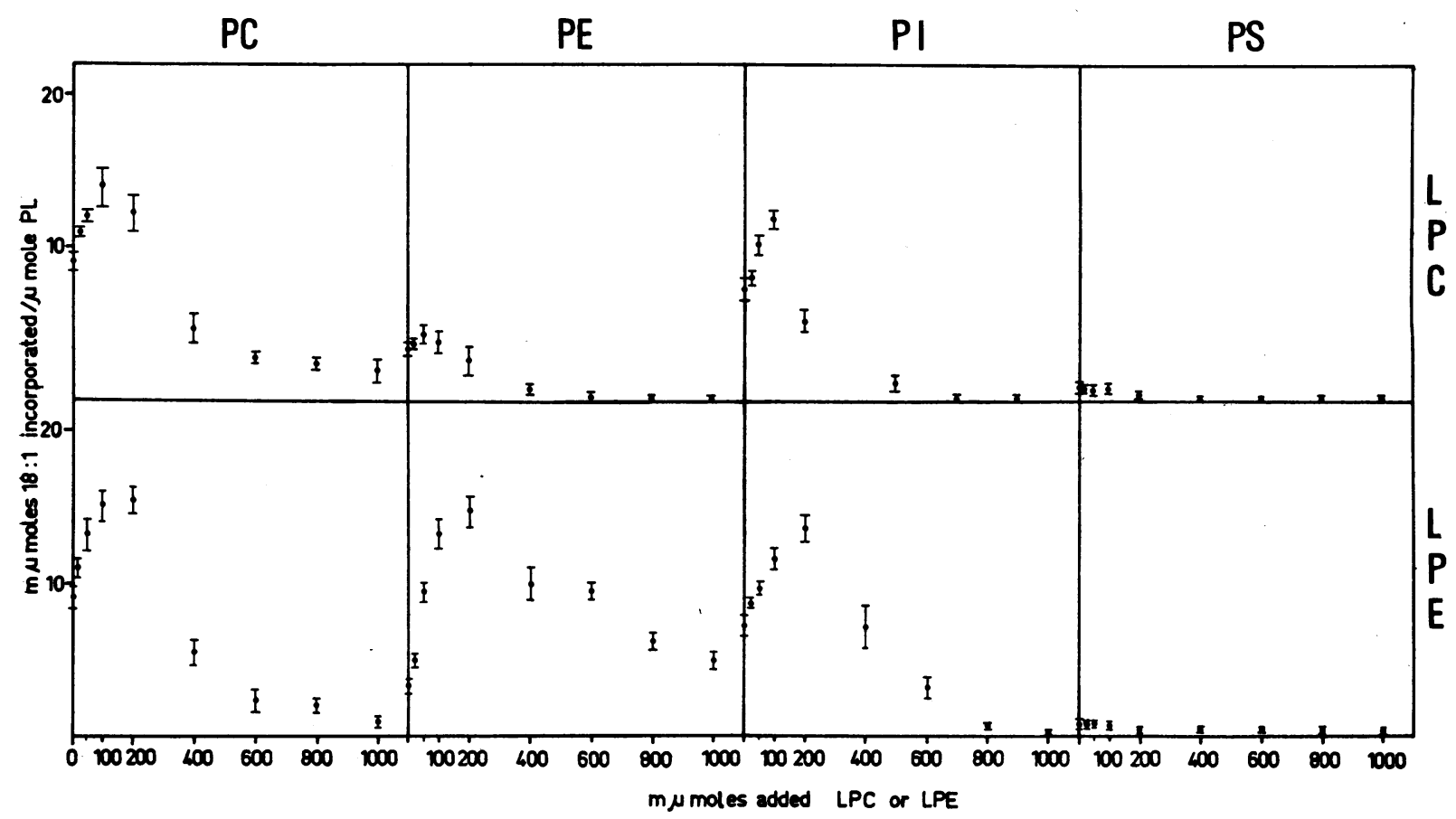

FIGURE 6 Effect of added LPC and LPE on patterns of incorporation of $1{ }^{14} \mathrm{C}-$ labeled 18:1 into major platelet PL. The ordinate shows the m $\mu$ moles 18:1 incorporated per $\mu$ mole PL; the abscissa, the amount of added LPC or LPE per incubation. In two experiments (not shown) the substitution of synthetic 1-16:0-LPC, for LPC derived from rat liver, gave similar results. The results shown are the means \pm SD of three experiments in which the effects of LPC and LPE were compared in parallel incubations on the same day using platelets derived from the same donor pair. 


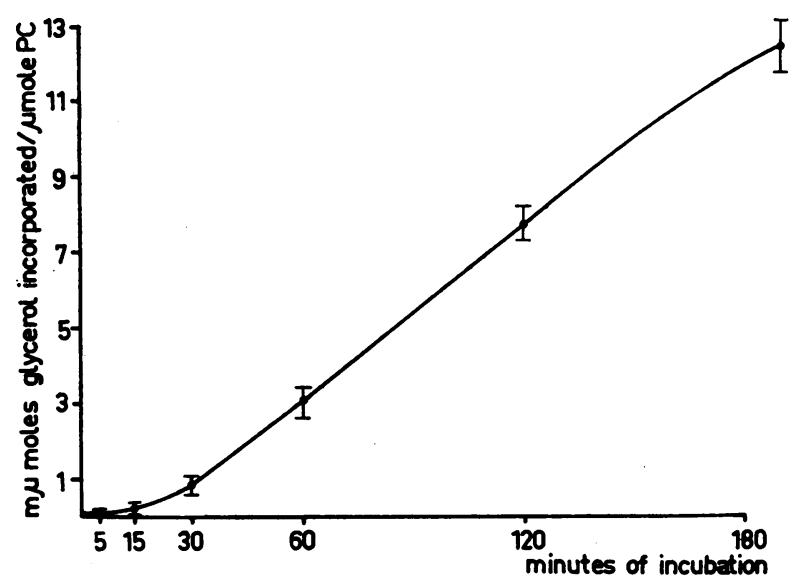

FIGURE 7 Incorporation of $2-{ }^{8} \mathrm{H}$-labeled glycerol into PC of human platelets. The abscissa shows the time of incubation; the ordinate, the m $\mu$ moles of glycerol incorporated per $\mu$ mole PC. The data shown are the means \pm SD of three experiments.

into $\mathrm{PC}$, by inference we established the presence of glycerol kinase (EC 2.7.1.30) in the platelet, since the de novo acylation of all lipids starts with G-3-P as a precursor (34).

The uptake of glycerol-2- ${ }^{8} \mathrm{H}$ into $\mathrm{PC}$ showed a lag phase of 15-30 min which was followed by a linear phase lasting $4 \mathrm{hr}$ (Fig. 7). Alkaline hydrolysis of the PC isolated after a $3 \mathrm{hr}$ incubation was done four times, with recovery of $52-77 \%$ of its radioactivity in GPC.

\section{DISCUSSION}

When incubated with human platelets, FA may be oxidized, or incorporated into NL and PL, the latter through the Lands (acylation of lyso derivatives) and probably the Kennedy (de novo synthesis) pathways. Although much further work will be necessary in order to define control mechanisms governing the interrelationship of the several pathways, certain observations are worth emphasizing at this time.

\section{FA oxidation}

FA oxidation by human platelets has been previously documented (35). The bulk of our observations related to various conditions affecting FA oxidation by human platelets are the subject of a separate report. ${ }^{11}$ The chief importance of FA oxidation as it relates to the present study is that it accounts for nearly all of the nonrecoverable radioactivity in the lipid extract of an incubation.

\section{Partitioning of added FA among PL}

In incubations with intact platelets, PE, PI, and PS compared with PC, get an increasingly larger share of

\footnotetext{
${ }^{11}$ Cohen, P., and B. Wittels. Energy substrate metabolism in fresh and stored human platelets. J. Clin. Invest. 49: 119.
}

added FA with: (a) longer periods of incubation with a fixed amount of added 18:1 (Fig. 4, top) and (b) higher concentrations of 18:1 (Fig. 4, bottom). There are several possible explanations for these findings. If the four PL are renewed almost exclusively by the Lands pathway $(a)$ the acyltransferases for each could have different $K_{m}$ values for 18:1, or different stabilities during prolonged incubation at $37^{\circ} \mathrm{C}$ (Reitz et al. have, in fact, suggested both of these possibilities in studies with rat liver microsomes) $(36)$; $(b)$ the endogenous lysocompounds may not be equally available for acyltransferase activity at the start of the incubation. For example, LPC acyltransferases may be at the cell surface, and acyltransferases for the other PL more sequestered and thus dependent on membrane damage during incubation to become "unmasked." Or, a phospholipase A (EC 3.1.1.4) with a preference for $\mathrm{PE}$ as substrate $(32,37)$ may act during the incubation to continuously furnish a supply of LPE for PE synthesis, while the concentration of LPC falls and becomes rate limiting for PC synthesis.

If the PL are renewed by different pathways (Lands vs. Kennedy, for example) the rates of the various reactions leading to their final synthesis could differ among the four compounds. In fact, with respect to $\mathrm{PC}$ and $\mathrm{PE}$, van den Bosch (37) and van Golde (38) have found suggestive evidence for this concept in studies with rat liver homogenates, and slices, respectively.

With the available data, it is not clear which among these possibilities is the most likely alternative.

\section{Patterns of FA incorporation into platelet PL}

With intact platelets, the observed patterns of FA incorporation into PL (Tables I and II) could also be related to various factors: (a) differences in transportpermeability behavior of the several FA; for example, the rates of the reactions catalyzed by specific acyl synthetases could differ, as has been shown in the studies of Pande and Mead with rat liver $(39) ;(b)$ the domination of the isomers of lyso derivatives of the various PL by those compounds which have a strong preference for certain reaction partners, as shown in the studies of Lands and Merkl with rat liver (9); and (c) specificity of acyltransferases responsible for esterification of FA to PL precursors; data in support of this possibility have also been reported from Lands' laboratory (26).

In keeping with the views of Lands et al. (26) we feel that specificity of acyltransferases plays the most important role, and that the in vitro data offer a fair degree of predictability for the observed patterns of FA distribution in the natural PL. In all but three instances, the relative incorporation of tracer amounts of a particular FA for certain PL was consistent with the relative concentrations of the FA in the PL (Table II). Ex- 
ceptions to this pattern were found where the FA represented a minor component of the PL. In these cases (18:2 for PE and PI, and 16:0 for PI) the $30 \mathrm{~m} \mu$ moles of added tracer constituted a very large fraction of the total amount of the FA species which was present in the incubation. Thus, any preference for particular FA as reaction partners may have been overruled by a limited choice of substrate.

\section{Effect of mechanical disruption of platelets on the} patterns of FA incorporation into PL

Despite the elimination of FA oxidation, with the consequent maintenance of higher substrate levels of FA, sonicated, as opposed to intact, platelets showed a reduced incorporation of $16: 0$ and 18:0 into $P C$ and $\mathrm{PE}$, whereas the opposite effect was found in parallel studies using 18:1 and 18:2. This suggests the possibility that sonication selectively damages acylsynthetase and (or) acyltransferase systems for the saturated, as opposed to the unsaturated, 16-18 carbon FA.

There are experimental data to support both of these possibilities. In their studies of rat liver microsomes Pande and Mead have found evidence for the presence of two different long-chain acyl CoA synthetases (EC 6.2.1.2), one for saturated and the other for unsaturated FA (39). And, as previously cited, Lands and coworkers have repeatedly suggested that a number of highly specific acyltransferases are involved in lipid synthesis $(26,29,36)$. Recently, in fact, Reitz et al. have furnished data to suggest that each fatty acyl-CoA may have its own acyltransferase (36).

\section{Incorporation of FA into NL}

With regard to the NL, this study confirms earlier observations of Deykin and Desser (6), that platelets are capable of incorporating FA into both DG and TG. However, our data for the postincubation distribution of added $16: 0-1-{ }^{14} \mathrm{C}$ among platelet lipids differs from that reported by Deykin and Desser (6). This discrepancy is possibly related to different conditions of incubation chosen by the two laboratories. Also, we did not detect labeling of the ceramide fraction. This is not likely to be due to different incubation conditions, but rather to our failure to vary chromatographic systems to effect a clear separation of this lipid, as was so elegantly achieved by those authors.

\section{Exploration of pathways of incorporation of FA} into PL

Lands pathway. It is difficult to separate the lytic properties of lyso compounds from their role as reaction partners for FA. And it is possible that a slight degree of membrane damage to all platelets, or a large degree of damage to some, occurs at very low concentrations of added lyso derivatives, since the lytic effect of the latter probably has a distribution curve among the total cell population (40). We did not exclude these possibilities, although gross thrombocytolysis was observed only at concentrations of LPC (or LPE) which were 25-50 times those shown in Table III.

The double-label experiments suggest that at concentrations of up to $30 \mathrm{~m} \mu$ moles of LPC per incubation, it functions chiefly as a reaction partner for the $\mathrm{FA}$, and not merely as an agency for "opening up holes" in the membrane to facilitate the transport or contact of FA with acyltransferase systems (Table III). Had the LPC acted mainly as a pathfinder for added FA, the latter would have shown a significant excess of incorporation into PC. In fact, the reverse appeared to be true, which could be interpreted as indicating the presence of a transacylation reaction (condensation of two molecules of LPC to form one molecule of PC, and one of GPC) $(37,41-43)$.

In the absence of ATP and CoASH, large amounts of LPC (greater than $50 \mu$ moles/liter) no longer stimulate, but actually reduce 18:1 incorporation into $\mathrm{PC}$. ATP and CoASH not only induce larger increments of stimulation at low concentrations of added LPC, but are able to sustain the acyltransferase reaction even in the presence of strongly lytic concentrations of LPC (Fig. 5). It is likely that, as has been found with erythrocytes, in the absence of exogenous ATP and CoASH, lysophospholipase activity predominates over the acylation reaction (44), furnishing concentrations of fatty acids which could alone, or in combination with excess LPC, interfere with acyltransferase activity.

It is also apparent that in the absence of ATP and CoASH, LPC and LPE are able to stimulate incorporation of 18:1 into $\mathrm{PE}$ and $\mathrm{PI}$, and $\mathrm{PC}$ and $\mathrm{PI}$, respectively (Fig. 6). This suggests that even at low concentrations of added lysoderivatives these compounds may exert a physicochemical effect on the membrane which enhances contact of the FA with several acyltransferase systems. This physicochemical effect apparently is augmented in the absence of optimal conditions for acyltransferase activity with a favored reaction partner. The fact that incorporation of $18: 1$ into PS is not similarly affected cannot be explained with the available data.

De novo pathway. That a de novo pathway of $\mathrm{PL}$ synthesis exists in human platelets was shown by the recovery of the ${ }^{8} \mathrm{H}$ label in GPC after alkaline hydrolysis of $\mathrm{PC}$ isolated from platelets which had been incubated for $3 \mathrm{hr}$ with glycerol-2- ${ }^{8} \mathrm{H}$. It is most likely that the operation of the pathway described by Kennedy and Weiss (10) for direct synthesis of PC accounts for these findings; however, methylation of labeled PE (45) is one of the possible alternatives to consider.

Fatty Acid Metabolism in Human Platelets 


\section{ACKNOWLEDGMENTS}

Through the cooperation of Dr. C. Dudok de Wit, the platelet procurement was done in his laboratory, the Blood Transfusion Service of the University Hospital, Utrecht, Holland. The technical assistance of Gysberta Broekema and Janet Shipman is gratefully acknowledged. We thank Professor L. L. M. van Deenen for numerous useful discussions and encouragement during the course of the work.

This study was supported in part by grants from: U. S. Public Health Service (7-F3-HE-6417-01A1); the General Research Support Fund of the Peter Bent Brigham Hospital, Boston, Mass.; the American Heart Association (67553) ; Baxter Laboratories, Morton Grove, Ill.; and the Netherlands Foundation for Chemical Research.

\section{REFERENCES}

1. Oliveira, M. M., and M. Vaughan. 1964. Incorporation of fatty acids into phospholipids of erythrocyte membranes. J. Lipid. Res. 5: 156.

2. Robertson, A. F., and W. E. M. Lands. 1964. Metabolism of phospholipids in normal and spherocytic human erythrocytes. J. Lipid Res. 5: 88.

3. Mulder, E., and L. L. M. van Deenen. 1965. Metabolism of red-cell lipids. I. Incorporation in vitro of fatty acids into phospholipids from mature erythrocytes. Biochim. Biophys. Acta. 106: 106.

4. Mulder, E., and L. L. M. van Deenen. 1965. Metabolism of red-cell lipids. III. Pathways for phospholipid renewal. Biochim. Biophys. Acta. 106: 348.

5. Shohet, S. B., D. G. Nathan, and M. L. Karnovsky. 1968. Stages in the incorporation of fatty acids into red blood cells. J. Clin. Invest. 47: 1096.

6. Deykin, D., and R. K. Desser. 1968. The incorporation of acetate and palmitate into lipids by human platelets. J. Clin. Invest. $47: 1590$.

7. Cohen, P., M. H. Cooley, and F. H. Gardner. 1965. Platelet preservation. III. Comparison of radioactivity yields of platelet concentrates derived from blood anticoagulated with EDTA and ACD. N. Engl. J. Med. 273: 845.

8. Lands, W. E. M. 1960. Metabolism of glycerolipids. II. The enzymatic acylation of lysolecithin. J. Biol. Chem. 235: 2233 .

9. Lands, W. E. M., and I. Merk1. 1963. Metabolism of glycerolipids. III. Reactivity of various acyl esters of coenzyme A with $\alpha^{\prime}$-acylglycerophosphorylcholine, and positional specificities in lecithin synthesis. J. Biol. Chem. 238: 898.

10. Kennedy, E. P., and S. B. Weiss. 1956. The function of cytidine coenzymes in the biosynthesis of phospholipids. J. Biol. Chem. 222: 193.

11. Brecher, G., and E. P. Cronkite. 1950. Morphology and enumeration of human blood platelets. J. Appl. Physiol. 3: 365 .

12. Gornall, A. G., C. S. Bardawill, and M. M. David. 1949. Determination of serum proteins by means of the biuret reaction. J. Biol. Chem. 177: 751.

13. van den Bosch, H., and L. L. M. van Deenen. 1965. Synthesis of ${ }^{22} \mathrm{P}-,{ }^{14} \mathrm{C}$-, and ${ }^{8} \mathrm{H}$-labeled lecithins and their use in studies on lipid metabolism. In Advances in Tracer Methodology. S. Rothschild, editor. Plenum Press Inc., New York. 3: 61 .

14. Bruno, G. A., and J. E. Christian. 1961. Correction for quenching associated with liquid scintillation counting. Anal. Chem. 33: 650.
15. van Deenen, L. L. M., and G. H. de Haas. 1963. The substrate specificity of phospholipase A. Biochim. Biophys. Acta. 70: 538.

16. Bartlett, G. R. 1955. Phosphorus assay in column chromatography. J. Biol. Chem. 234: 466.

17. Baer, E., and D. Buchnea. 1959. Synthesis of saturated and unsaturated L- $\alpha$-lecithins. Acylation of the cadmium chloride compounds of L- $\alpha$-glycerolphosphorylcholine. Can. J. Biochem. Physiol. 37: 953.

18. Bergmeyer, H., and E. Bernt. 1965. Methods of Enzymatic Analysis. H. Bergmeyer, editor. Academic Press Inc., New York. 266-270.

19. Bligh, E. G., and W. J. Dyer. 1959. A rapid method of total lipid extraction and purification. Can. J. Biochem. Physiol. 37 : 911.

20. Ways, P., and D. J. Hanahan. 1964. Characterization and quantification of red cell lipids in normal man. J. Lipid Res. 5: 318 .

21. Skipsky, V. P., R. F. Peterson, and M. Barclay. 1964. Quantitative analysis of phospholipids by thin-layer chromatography. Biochem. J. 90: 374 .

22. Hofmann, A. F. 1963. Thin-layer chromatography of lipids. In Biochemical Problems in Lipids. A. C. Frazer, editor. Elsevier, Amsterdam. 1-16.

23. Owens, K. 1966. A two-dimensional thin-layer chromatographic procedure for the estimation of plasmalogens. Biochem. J. 100: 354.

24. Benson, A. A., and B. Maruo. 1958. Plant phospholipids. I. Identification of the phosphatidyl glycerols. Biochim. Biophys. Acta. 27 : 189.

25. Snyder, F., and P. Godfrey. 1961. Collecting ${ }^{14} \mathrm{CO}_{2}$ in a Warburg flask for subsequent scintillation counting. J. Lipid Res. 2: 195.

26. Lands, W. E. M., M. L. Blank, L. J. Nutter, and O. Privett. 1966. A comparison of acyltransferase activities in vitro with the distribution of fatty acids in lecithins and triglycerides in vivo. Lipids. 1: 224.

27. Cohen, P., and A. Derksen. 1969. Comparison of phospholipid and fatty acid composition of human erythrocytes and platelets. Brit. J. Haematol. 17: 159.

28. Veerkamp, J. H., I. Mulder, and L. L. M. van Deenen. 1962. Comparison of the fatty acid composition of lipids from different animal tissues including some tumours. Biochim. Biophys. Acta. 57: 299.

29. Lands, W. E. M., and P. Hart. 1965. Metabolism of glycerolipids. VI. Specificities of acyl coenzyme A: phospholipid acyltransferases. J. Biol. Chem. 240: 1095.

30. Lehninger, A. L. 1964. The Mitochondrion. W. A. Benjamin Inc., New York. 151.

31. Tzur, R., E. Tal, and B. Shapiro. 1964. $\alpha$-Glycerophosphate as regulatory factor in fatty acid esterification. Biochim. Biophys. Acta. 84: 18.

32. Scherphof, G. L. 1967. Metabolic conversions of mitochoindrial and microsomal phospholipids. Ph.D. Thesis, University of Utrecht.

33. Cooley, M. H., and P. Cohen. 1967. Potassium transport in human blood platelets. J. Lab. Clin. Med. 70: 69 .

34. Kornberg, A., and W. E. Pricer, Jr. 1953. Enzymatic esterification of $\alpha$-glycerophosphate by long-chain fatty acids. J. Biol. Chem. 204: 345.

35. Rosenzweig, A., and P. Ways. 1966. The oxidation of long-chain fatty acids by the formed elements of human blood. Blood. 27 : 57.

36. Reitz, R. C., M. El-Sheikh, W. E. M. Lands, I. A. Ismail, and F. D. Gunstone. 1969. Effects of ethylenic 
bond position upon acyltransferase activity with isomeric cis-octadecanoyl coenzyme A thiol esters. Biochim. Biophys. Acta. 176: 480.

37. van den Bosch, H. 1966. Lysolecithins, their enzymatic formation and conversions. Ph.D. Thesis, University of Utrecht.

38. van Golde, L. M. G. 1968. Analysis and metabolism of molecular species of phospholipids. Ph.D. Thesis, University of Utrecht.

39. Pande, S. V., and J. F. Mead. 1968. Long-chain fatty acid activation in subcellular preparations from rat liver. J. Biol. Chem. 243: 352.

40. Collier, H. B. 1951. Factors affecting the hemolytic action of "lysolecithin" upon rabbit erythrocytes. J. Gen. Physiol. 35: 617.

41. Erbland, J. F., and G. V. Marinetti. 1962. In vitro metabolism of lysolecithin. Fed. Proc. 21 : 295. (Abstr.)
42. Kokke, R., G. J. M. Hooghwinkel, H. L. Booij, H. van den Bosch, L. Zelles, E. Mulder, and L. L. M. van Deenen. 1963. Metabolism of lysolecithin and lecithin in a yeast supernatant. Biochim. Biophys. Acta. 70: 351.

43. Elsbach, P. 1966. Phospholipid metabolism by phagocytic cells. I. Comparison of conversion of ${ }^{22} \mathrm{P}$ lysolecithin to lecithin and glycerophosphorylcholine by homogenates of rabbit polymorphonuclear leukocytes and alveolar macrophages. Biochim. Biophys. Acta. 125: 510.

44. Mulder, E., J. W. O. van den Berg, and L. L. M. van Deenen. 1965. Metabolism of red cell lipids. II. Conversions of lysophosphoglycerides. Biochim. Biophys. Acta. 106: 118.

45. Bremer, J. P., P. H. Figard, and D. M. Greenberg. 1960. The biosynthesis of choline and its relation to phospholipid metabolism. Biochim. Biophys. Acta. 43: 477. 